\title{
Evaluación de gel formulado a base de extracto hidroalcohólico del fruto de la papaya (Carica papaya $L$.) en el tratamiento de las úlceras por presión de estadío I y II".
}

\section{Evaluation of formulated gel based on hydroalcoholic extract of the fruit of the papaya (Carica papaya L.) in the treatment of ulcers by pressure of stage I and II}

\author{
Carlos Bell ${ }^{1}$
}

http://dx.doi.org/10.21503/CienciayDesarrollo.2013.v16i2.04

\section{RESUMEN}

Se evaluó el efecto de gel formulado a base de extracto hidroalcohólico del fruto de la papaya (Carica papaya $L$.), sobre las úlceras por presión a nivel de coxis, en 35 voluntarios de ambos sexos con edades comprendidas entre 50 y 70 años, todos residentes en la ciudad de Lima (Perú). La aplicación y seguimiento farmacológico se realizó una vez al día durante un mes y la medición de las heridas cada semana; la cantidad aplicada varió según la extensión y estadío de la úlcera; se excluyó la utilización de cualquier otro preparado convencional. El ensayo, previo consentimiento informado, mostró tener efecto en el proceso de cicatrización y regeneración del tejido comprometido por la úlcera por presión. El 60,00\% fue evaluado como excelente, el $25,00 \%$ como bueno y el $15,00 \%$ como regular. Los histogramas y gráficos lineales fueron trabajados en IBM SPSS 21, en los cuales se observa la dispersión de los datos y el comportamiento de la variable tamaño, que muestra un notable decrecimiento. Se concluye que el fruto de la papaya es una fuente de materia prima natural, de bajo costo, que eleva la calidad de atención en enfermería en el tratamiento de las úlceras por presión de estadío I y II.

Palabras Clave: Gel de papaya, úlceras por presión, cicatrización.

\section{ABSTRACT}

It was assessed the effect of formulated gel based on hydroalcoholic extract of the fruit of the papaya (Carica papaya L.) about ulcers by pressure in coccyx level, in 35 volunteers of both sexes, aged between 50 and 70 years, all residents in the city of Lima (Peru). The application and drug monitoring was performed once a day for a month and the measurement of wounds each week, the amount applied varied according to the size and stage of the ulcer, it was excluded the use any other conventional preparation. The trial, previous informed consent, it was shown to have effect on the healing process and tissue regeneration compromised by the pressure ulcer. The $60.00 \%$ was evaluated as excellent, $25.00 \%$ as good and $15.00 \%$ as fair. Histograms and line graph were worked in IBM SPSS 21, which shows the distribution of data and the behavior of the variable size, showing a remarkable decrease. We conclude that the fruit of the papaya is a natural source of raw material, low cost, which raises the quality of nursing care in the treatment of pressure ulcers by stage I and II.

Keywords: Papaya gel, pressure ulcers, healing.

1 Magister en Recursos Vegetales y Terapéuticos. Doctor en Farmacia y Bioquímica. Docente de la Universidad Nacional Mayor de San Marcos (UNMSM). Docente UAP. E-mail: carlosbellcortez@yahoo.es 


\section{INTRODUCCIÓN}

Una úlcera por presión (UPP) es una lesión de los tejidos que hay entre la epidermis y una prominencia ósea, debida principalmente a una fuerza de presión externa; esta presión puede estar producida por el propio peso del paciente sobre la superficie en que está apoyado (habitualmente sillón o cama) o por otros dispositivos terapéuticos, como sondas, mascarillas de oxígeno o férulas, entre otras ${ }^{(1)}$.La presión capilar media tiene valores aproximados de $17 \mathrm{mmHg}$; una presión superior mantenida sobre una zona concreta provoca aplastamiento del tejido y la consecuente oclusión capilar, con lo que el tejido se ve privado de oxígeno y de los nutrientes adecuados. La reducción sanguínea y los mecanismos de reperfusión natural que pone en marcha el organismo cuando se libera la presión (hiperemia reactiva), llevan a una acumulación de catabolitos tóxicos en el tejido, con el consiguiente aumento de la permeabilidad capilar, dilatación vascular, formación de edema e infiltración celular. Este tipo de reacciones inflamatorias desencadena una hiperemia, acompañada de un aumento de la presión capilar, por lo cual hasta este instante aún pueden evacuarse los catabolitos tóxicos y regenerarse las células de la piel, con la condición que se retire completamente la presión de la zona. Si esto no ocurre, la progresiva hipoxia (privación del suministro de oxígeno) produce una muerte irreversible de las células con la aparición de necrosis (muerte del tejido)(2). Son heridas de larga evolución que requieren largo tiempo para curar por su complejo proceso de cicatrización .Las zonas de mayor riesgo de desarrollo de UPP son la región sacra, los talones, las tuberosidades isquiáticas y las caderas; todas ellas con tejido subcutáneo insuficiente para amortiguar la presión ${ }^{(3)}$ . Las UPP son un importante problema de salud por multitud de motivos. Algunas heridas pueden implicar importantes consecuencias como las infecciones generalizadas o amputaciones en el caso de úlceras de pie diabético; son heridas de larga evolución, que requieren largos periodos de tiempo para curar (meses), por su complejo proceso de cicatrización que elimina y reemplaza el tejido dañado ${ }^{(4)}$. El costo que suponen deriva sobretodo de los recursos humanos para atender a estos pacientes, el material de curas (aproximadamente del uno al tres por ciento del coste total), el incremento de gasto por complicaciones asociadas y del aumento de las estancias hospitalarias

No cabe duda que las UPP influyen negativamente en las personas que las padecen, retrasando su rehabilitación y reinserción social, disminuyendo su autoestima e impactando en su calidad de vida. Más allá de las cifras que revelan costos económicos se debería considerar también el gran deterioro emocional y físico del propio paciente como el del ámbito familiar cercano que son difíciles de cuantificar y que afectan en relación directa la calidad de vida de los pacientes ${ }^{(5)}$.

El presente trabajo, basado en el conocimiento ancestral de emplear la pulpa de la papaya verde, en el tratamiento de las úlceras por presión, aspira a darle solidez científica a este conocimiento popular, empleando una especialidad farmacéutica en forma de gel, elaborado a base de un extracto hidroalcohólico de la papaya verde.

\section{Hipótesis}

El extracto hidroalcohólico del fruto de la papaya (Carica papaya L.), aplicado en forma de gel, tiene efecto regenerador y cicatrizante en los tejidos de las úlceras por presión de estadío I y II.

\section{MÉTODOS Y RESULTADOS}

\section{Material:}

A. Reactivos: Etanol absoluto, ácido poliacrílico, alcohol isopropílico, propilenglicol, Edta, preservantes, agua destilada estéril. 
B. Equipos: Espectrofotómetro UV-Visible, Marca Thermo Scientific, Modelo Helios-Zeta; Cromatógrafo de gases Perkin Elmer modelo Clarus 600; Espectrómetro Perkin Elmer modelo Spectrum 100N; Balanza Analítica Marca Ohaus Modelo Adventurer; Equipo emulsificador de laboratorio; Potenciómetro; Termómetro digital. Estufa Marca Labor Musze Ripari. Computador que soporte el análisis estadístico en IBM SPSS 21.

\section{METODOLOGÍA}

A. Clasificación taxonómica, recolección y preparación del extracto

\author{
REINO:Plantae \\ DIVISIÓN:Magnoliophyta \\ CLASE:Magnoliopsida \\ ORDEN:Brassicales \\ FAMILIA:Caricaceae \\ GÉNERO:Carica \\ ESPECIE: Carica papaya L.
}

Se seleccionaron frutos verdes de papaya (Carica papaya L.) de un huerto ubicado en el distrito de Chaclacayo (Lima-Perú).

Se procedió a lavarlas, secarlas y retirarle la cáscara; luego se cortaron en pedazos pequeños y se licuaron con alcohol etílico de $70^{\circ}$. Se dejó macerar por siete días en oscuridad para la extracción de los metabolitos. Luego, se filtró y se obtuvo una solución amarillenta que se evaporó en estufa de aire circulante a temperatura no superior a $40{ }^{\circ} \mathrm{C}$, obteniéndose un extracto pilular de color marrón.

\section{B. Análisis físico químico del extracto de papa- ya (Carica papaya L.)}

Se efectuaron los siguientes análisis físicos: aspecto, color olor, sabor, $\mathrm{pH}$, humedad ${ }^{(6)}$.

Análisis químico: cenizas.

\section{Análisis instrumental del extracto de papaya (Carica papaya L.)}

Se determinó el espectro NIRS al extracto.

\section{Formulación y estabilidad del gel}

Se ensayó la formulación que se describe, para lo cual se tuvo en cuenta que los ingredientes seleccionados sean compatibles con el extracto obtenido y que sobretodo, sean estables en el tiempo así como de bajo costo:

Extracto de Papaya. $30,00 \mathrm{~g}$

Agentes gelificantes, diluyentes y

preservantes. $.7,70 \mathrm{~g}$

Agua destilada estéril c.s.p. $100,00 \mathrm{~g}$

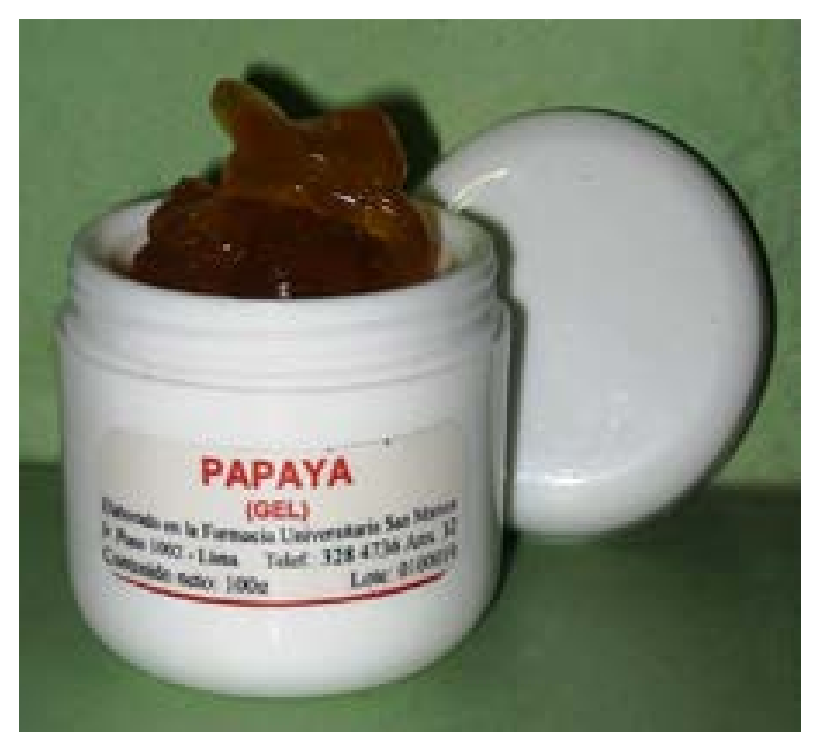

Figura 1. Gel formulado con papaya

Ensayo de estabilidad acelerada de la formulación.

Con el fin de determinar la estabilidad del preparado y controlar sus posibles alteraciones, se efectuaron ensayos de estabilidad acelerada, sometiendo la formulación a diferentes temperaturas. Para realizar esta prueba, se procedió a evaluar cada quince días, hasta completar tres meses, los siguientes parámetros: aspecto, consistencia, color, olor y $\mathrm{pH}$. 


\section{G. Ensayo efectuado en voluntarios}

Participaron en el estudio, 25 voluntarios con úlceras por presión de estadío I y II de ambos sexos, con edades comprendidas entre 50 y 70 años, residentes de diversos distritos de Lima Metropolitana.

Se dividió a los participantes en dos grupos:

- Grupo I (grupo problema-30 pacientes): Se administró gel con extracto de papaya.

- Grupo II (grupo control-5 pacientes): Se administró gel sin extracto de papaya.

Previo consentimiento escrito, se procedió a la aplicación del gel a base de extracto de papaya a los pacientes voluntarios, en la úlcera por presión una vez al día, practicándose previamente un lavado con suero fisiológico, la úlcera fue cubierta con papel plastificado para otorgar un ambiente húmedo a la zona y finalmente se cubrió con gasa toda la extensión de la úlcera por presión fijándola con esparadrapo.
El estudio se extendió durante 30 días y se administró una dosis de preparado que dependió del tamaño de la herida, excluyendo la utilización de cualquier otro preparado convencional. El seguimiento farmacológico se efectúo todos los días.

\section{H. Análisis estadístico}

Se utilizó el programa IBM SPSS 21, en el cual se creó una base de datos con las mediciones obtenidas de las 4 semanas, en las cuales evidentemente se observó la reducción del tamaño del la úlcera. El programa analizó el comportamiento de nuestra variable escalar (tamaño) durante las cuatro semanas y asimismo por medio de los histogramas se obtuvo la dispersión de los datos para observar la evolución de toda la población (30 pacientes). Los 15 primeros datos pertenecen a pacientes con ulceras de grado I y los 15 siguientes ulceras de grado II

Tabla 1. Mediciones en centímetros obtenidas de las úlceras de los pacientes por semana

\begin{tabular}{|cccc|}
\hline Semana 1 & Semana & Semana & Semana 4 \\
\hline 2 & 1,5 & 0,5 & 0 \\
\hline 1,5 & 1 & 0,2 & 0 \\
3 & 2,2 & 1,1 & 0 \\
\hline 2,5 & 1,8 & 0,9 & 0 \\
\hline 2,2 & 1,5 & 0,7 & 0 \\
\hline 3,2 & 2,5 & 1,1 & 0,2 \\
\hline 2,5 & 1,2 & 0,6 & 0 \\
1,4 & 0,8 & 0,1 & 0 \\
\hline 2,3 & 1,6 & 0,9 & 0 \\
\hline 1,5 & 1 & 0,3 & 0 \\
\hline 1,2 & 0,8 & 0,3 & 0 \\
\hline 2,5 & 1,4 & 0,5 & 0 \\
\hline 3,6 & 2,5 & 1 & 0 \\
\hline 2,7 & 1,9 & 0,8 & 0 \\
\hline 3,2 & 2,2 & 0,9 & 0 \\
\hline 6,3 & 5,4 & 3,9 & 2,3 \\
\hline 5,3 & 3,8 & 2,1 & 0,5 \\
\hline 4,8 & 3,7 & 1,9 & 0,3 \\
\hline 5,1 & 3,9 & 2,3 & 0,8 \\
\hline
\end{tabular}




\begin{tabular}{lccc}
4,2 & 3,1 & 2,3 & 1,1 \\
5,2 & 4 & 3 & 1,9 \\
4,7 & 3,6 & 2,5 & 1,3 \\
4,9 & 3,8 & 2,6 & 1,4 \\
6,3 & 5,1 & 3,2 & 1,1 \\
5,6 & 4,2 & 2,6 & 0,8 \\
4,8 & 3,6 & 2 & 0,6 \\
\hline 5,2 & 3,9 & 2,4 & 0,7 \\
4,6 & 3,2 & 1,9 & 0,6 \\
4,1 & 2,9 & 1,4 & 0,3 \\
\hline 5,4 & 4,1 & 2,3 & 0,8 \\
\hline
\end{tabular}

\section{RESULTADOS}

Marcha fitoquimica del extracto hidroalcohólico de papaya (Carica papaya L.)

Se determinó la presencia de:

- Flavonoides

- Taninos

- Triterpenoides

A. Análisis físico químico del extracto hidroalcohólico de papaya (Carica papaya L.)

Tabla 2. Protocolo de análisis

\section{PROTOCOLO DE ANÁLISIS DEL EXTRACTO DE PAPAYA}

\begin{tabular}{ll}
$\begin{array}{l}\text { PRODUCTO } \\
\text { CANTIDAD }\end{array}$ & $\begin{array}{l}: \text { Extracto de Papaya (Carica papaya L.) } \\
: 50 \mathrm{~g}\end{array}$ \\
$\begin{array}{l}\text { FECHA de Preparación de Extracto } \\
\text { FECHA DE ANÁLISIS }\end{array}$ & $\begin{array}{l}\text { Abril de } 2013 \\
\text { Abril de } 2013\end{array}$ \\
DETERMINACIONES & RESULTADOS \\
A) FÍSICAS: & \\
ASPECTO & Masa blanda \\
COLOR & Marrón claro \\
SABOR & Ligeramente dulce \\
OLOR & Sui géneris. \\
pH (Solución acuosa $\left.10 \%-25^{\circ} \mathrm{C}\right)$ & 2,50 \\
HUMEDAD & $3 . .17 \%$ \\
B) QUÍMICAS: & \\
CENIZAS TOTALES & $2.88 \%$ \\
\hline
\end{tabular}




\section{B. ANÁLISIS POR INSTRUMENTACIÓN DE Carica papaya L.}

Espectro NIRS de Papaya $\left(1 / \mathrm{R}, \mathrm{cm}^{-1}\right)$

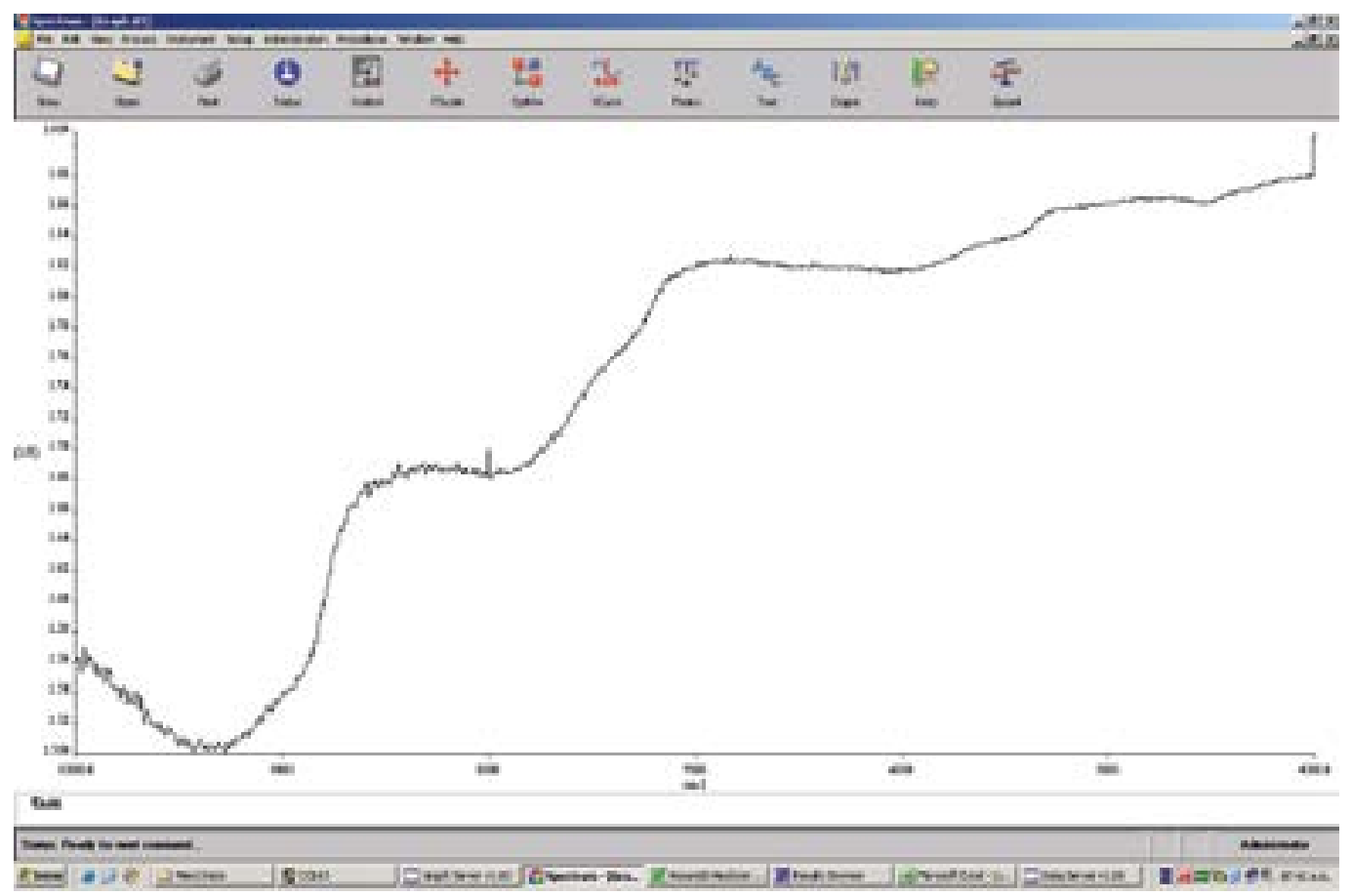

Espectro NIRS de Papaya (\%T, $\left.\mathrm{cm}^{-1}\right)$

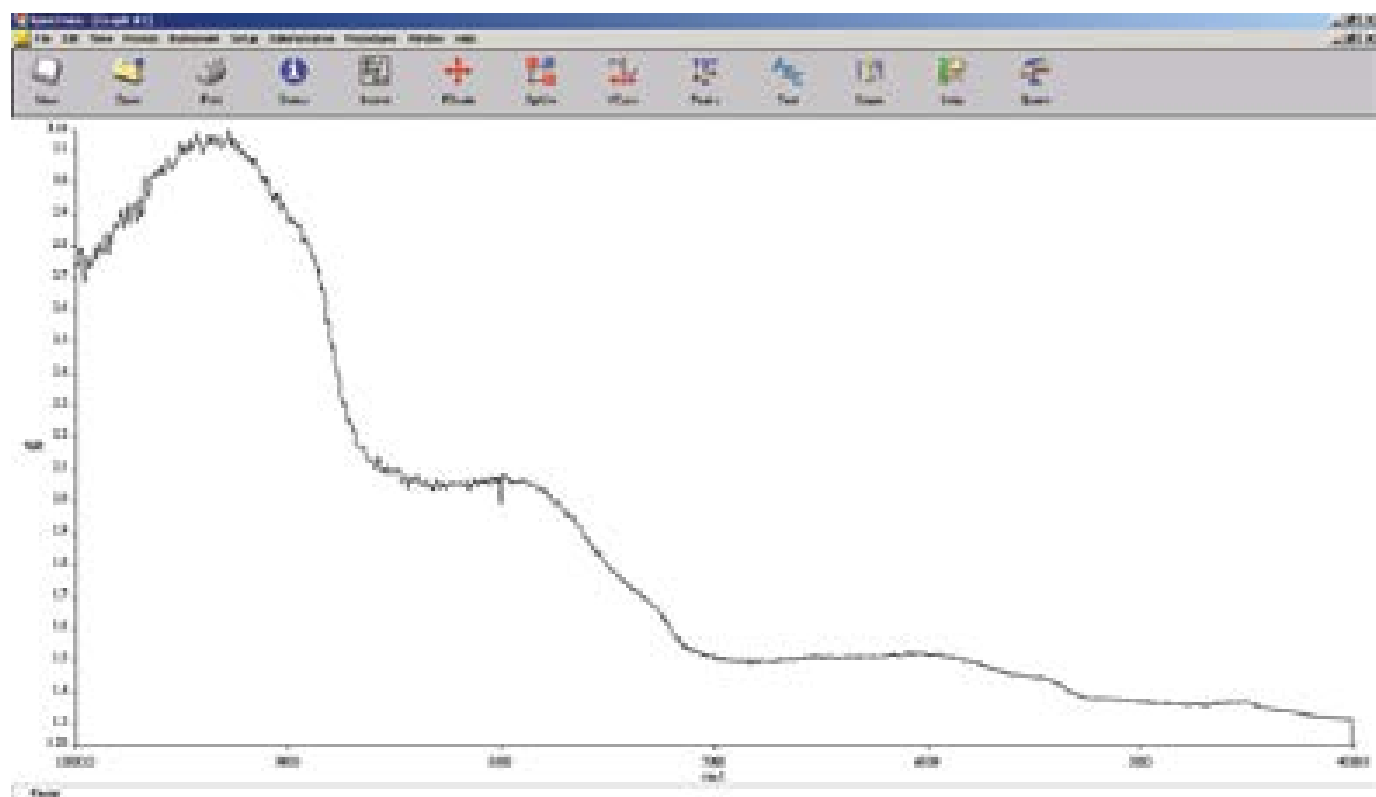

$\rightarrow$

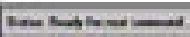

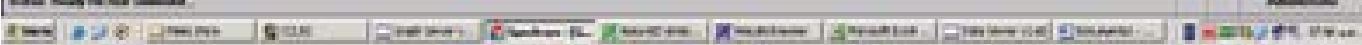




\section{EVALUACIÓN FARMACOTÉCNICA DEL GEL}

\section{FICHA TÉCNICA DE ESTABILIDAD DEL PRODUCTO TERMINADO}

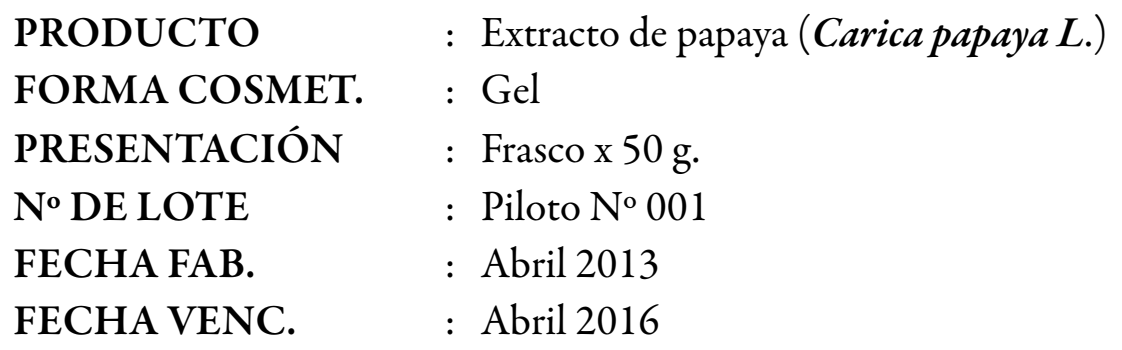

\section{RESULTADOS}

Tabla 3. Estabilidad

\begin{tabular}{|c|c|c|c|c|c|}
\hline \multirow{2}{*}{ DETERMINACIONES } & \multirow{2}{*}{ ESPECIFICACIONES } & \multicolumn{4}{|c|}{ RESULTADOS } \\
\hline & & Inicio & $1 \mathrm{mes}$ & 2 meses & 3 meses \\
\hline ASPECTO & Gel translúcido & Cumple & Cumple & Cumple & Cumple \\
\hline COLOR & Marrón anaranjado & Cumple & Cumple & Cumple & Cumple \\
\hline OLOR & Sui géneris & Cumple & Cumple & Cumple & Cumple \\
\hline VISCOSIDAD & $100000-150000 \mathrm{cps}$ & 130000 & 120000 & 120000 & 120000 \\
\hline $\mathrm{pH}$ & $5,5-7,0$ & 5,9 & 6,0 & 6,0 & 6,1 \\
\hline \multicolumn{6}{|l|}{$\begin{array}{l}\text { RECUENTO } \\
\text { MICROBIANO }\end{array}$} \\
\hline $\begin{array}{l}\text { AEROBIOS } \\
\text { MESOFILOS }\end{array}$ & Menos de $100 \mathrm{ufc} / \mathrm{g}$ & Conforme & Conforme & Conforme & Conforme \\
\hline $\begin{array}{l}\text { RECUENTO DE } \\
\text { HONGOS }\end{array}$ & Menos de $10 \mathrm{ufc/g}$ & Conforme & Conforme & Conforme & Conforme \\
\hline $\begin{array}{l}\text { RECUENTO DE } \\
\text { LEVADURAS }\end{array}$ & Menos de $10 \mathrm{ufc} / \mathrm{g}$ & Conforme & Conforme & Conforme & Conforme \\
\hline ESCHERICHIA COLI & Ausente en $10 \mathrm{~g}$ & Conforme & Conforme & Conforme & Conforme \\
\hline SALMONELLA spp & Ausente en $10 \mathrm{~g}$ & Conforme & Conforme & Conforme & Conforme \\
\hline $\begin{array}{l}\text { STAPHYLOCOCCUS } \\
\text { AUREUS }\end{array}$ & Ausente en $10 \mathrm{~g}$ & Conforme & Conforme & Conforme & Conforme \\
\hline $\begin{array}{l}\text { PSEUDOMONAS } \\
\text { AERUGINOSA }\end{array}$ & Ausente en $10 \mathrm{~g}$ & Conforme & Conforme & Conforme & Conforme \\
\hline
\end{tabular}




\section{EVALUACIÓN FARMACOLÓGICA}

Tabla 4. Valoración Farmacológica por semanas

\begin{tabular}{|c|c|c|c|c|c|c|c|c|c|c|c|c|c|c|c|c|}
\hline & \multicolumn{4}{|c|}{$1^{\circ}$ Semana } & \multicolumn{4}{|c|}{$2^{\circ}$ Semana } & \multicolumn{4}{|c|}{$3^{\circ}$ Semana } & \multicolumn{4}{|c|}{$4^{\circ}$ Semana } \\
\hline & GR & JPO I & GR & UPO I] & I GRI & UPO I & GR & UPO I & I GRI & UPO I & GRI & UPO I & II GRI & UPO I & GRL & JPO II \\
\hline & $\mathrm{N}^{\circ}$ & $\%$ & $\mathrm{~N}^{\circ}$ & $\%$ & $\mathrm{~N}^{\circ}$ & $\%$ & $\mathrm{~N}^{\circ}$ & $\%$ & $\mathrm{~N}^{\circ}$ & $\%$ & $\mathrm{~N}^{\circ}$ & $\%$ & $\mathrm{~N}^{\circ}$ & $\%$ & $\mathrm{~N}^{\circ}$ & $\%$ \\
\hline Excelentes & - & - & - & - & - & - & - & - & 7 & 35,00 & - & - & 12 & 60,00 & - & - \\
\hline Buenos & - & - & - & - & 9 & 45,00 & - & - & 8 & 40,00 & - & - & 5 & 25,00 & - & - \\
\hline Regulares & 16 & 80,00 & - & - & 10 & 50,00 & - & - & 5 & 25,00 & - & - & 3 & 15,00 & - & - \\
\hline Nulo & 4 & 20,00 & 5 & 100,0 & 1 & 5,00 & 5 & 100,0 & - & - & 5 & 100,0 & - & - & 51 & 100,0 \\
\hline Total & 20 & 100,0 & 5 & 100,0 & 20 & 100,0 & 5 & 100,0 & 20 & 100,0 & 5 & 100,0 & 20 & 100,0 & 51 & 100,0 \\
\hline
\end{tabular}

De acuerdo con la tabla calificadora de resultados de Balbuena Gonzáles y Chapano Jairo (1987) la evaluación clínica corresponde a la clasificación que se señala en la tab No 5

Tabla 5. Calificación de resultados

\begin{tabular}{lcc} 
CALIFICACIÓN & N $^{\circ}$ casos & $\%$ \\
\hline $\begin{array}{l}\text { EXCELENTES } \\
\text { (reducción entre 80-100\%) }\end{array}$ & 18 & 60,00 \\
$\begin{array}{l}\text { BUENOS } \\
\text { (reducción entre el 60-80\%) }\end{array}$ & 7 & 25,00 \\
$\begin{array}{l}\text { REGULARES } \\
\text { (reducción entre 40-60\%) }\end{array}$ & 5 & 15,00 \\
$\begin{array}{l}\text { NULO } \\
\text { (reducción menor de un 40\%) }\end{array}$ & - & - \\
$\begin{array}{l}\text { SUB - TOTAL } \\
\text { “GRUPO CONTROL.” }\end{array}$ & 30 & 100 \\
\hline TOTALES & 5 & \\
\hline
\end{tabular}

\section{E. RESULTADOS ESTADÍSTICOS}

Al realizar los análisis estadísticos de los datos se obtuvo la siguiente tabla, en donde podemos apreciar entre ellos a la moda, el mínimo y máximo valor de los datos por semana. 
Tabla 6. Resultados de análisis estadístico por semanas

\begin{tabular}{|c|c|c|c|c|c|}
\hline \multicolumn{6}{|c|}{ ESTADÍSTICOS } \\
\hline \multirow{3}{*}{$\mathbf{N}$} & & Semana 1 & Semana 2 & Semana 3 & Semana 4 \\
\hline & Válidos & 30 & 30 & 30 & 30 \\
\hline & Perdidos & 0 & 0 & 0 & 0 \\
\hline Media & & 3,7267 & 2,7400 & 1,5433 & 0,4900 \\
\hline Mediana & & 3,8500 & 2,7000 & 1,2500 & 0,2500 \\
\hline Moda & & 2,50 & 0,80 & 0,90 & 0,00 \\
\hline Desv. Tip. & & 1,55074 & 1,31742 & 1,01800 & 0,62607 \\
\hline Varianza & & 2,405 & 1,736 & 1,036 & 0,392 \\
\hline Mínimo & & 1,20 & 0,80 & 0,10 & 0,00 \\
\hline \multirow[t]{2}{*}{ Máximo } & & 6,30 & 5,40 & 3,90 & 2,30 \\
\hline & 25 & 2,4500 & 1,5000 & 0,6750 & 0,0000 \\
\hline \multirow[t]{2}{*}{ Percentiles } & 50 & 3,8500 & 2,7000 & 1,2500 & 0,2500 \\
\hline & 75 & 5,1250 & 3,8250 & 2,3250 & 0,8000 \\
\hline
\end{tabular}

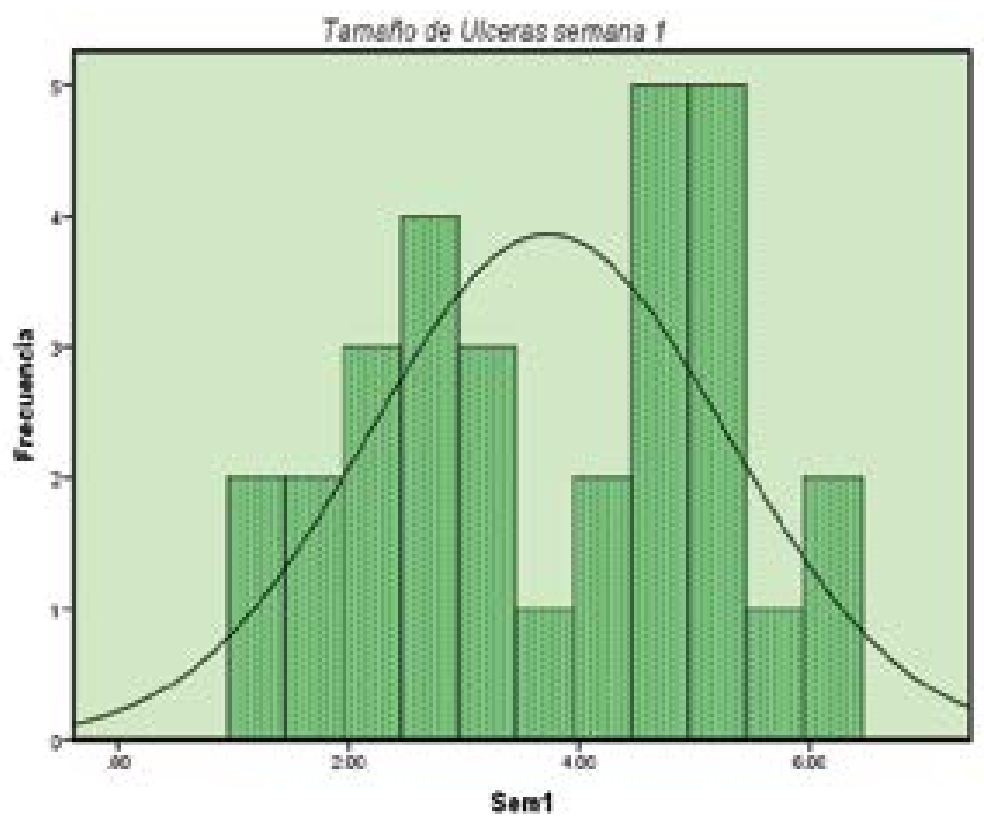

Figura 2. Histograma de distribución de los datos obtenidos de las mediciones realizadas la primera semana

En la semana 1 observamos una distribución de datos heterogénea, debido a que las úlceras tratadas de grado I y II poseían diferentes tamaños. 


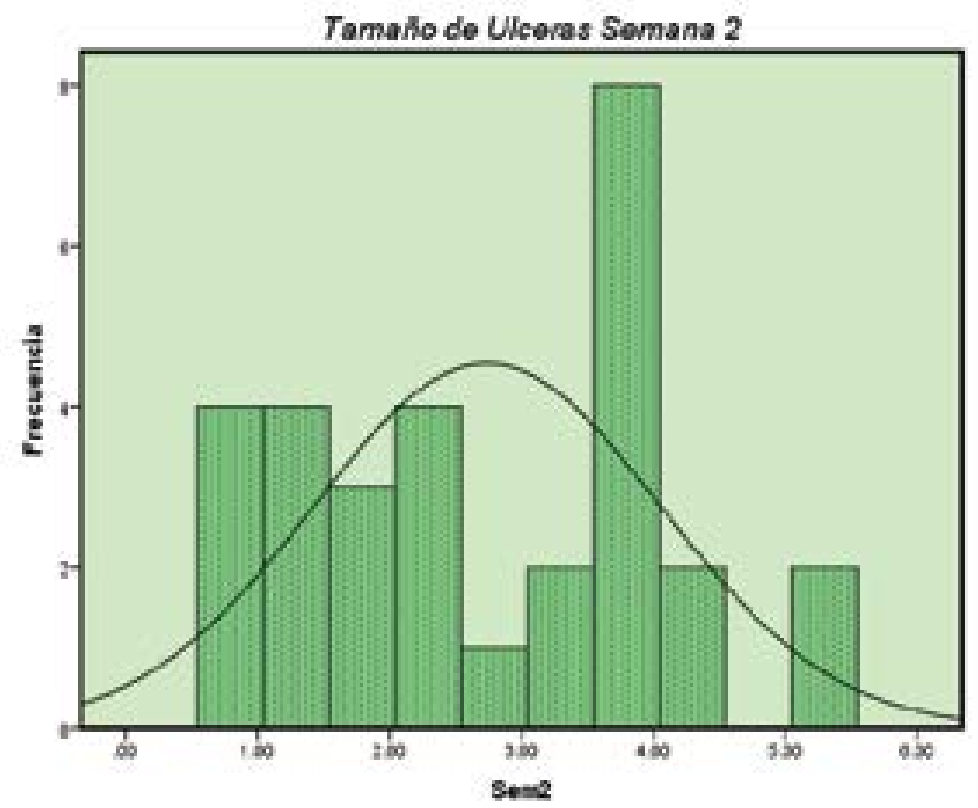

Figura 3. Histograma de distribución de los datos obtenidos de las mediciones realizadas la segunda semana

En la semana 2 observamos el ordenamiento de los datos hacia la izquierda lo cual nos indica que las ulceras están decreciendo debido al tratamiento.

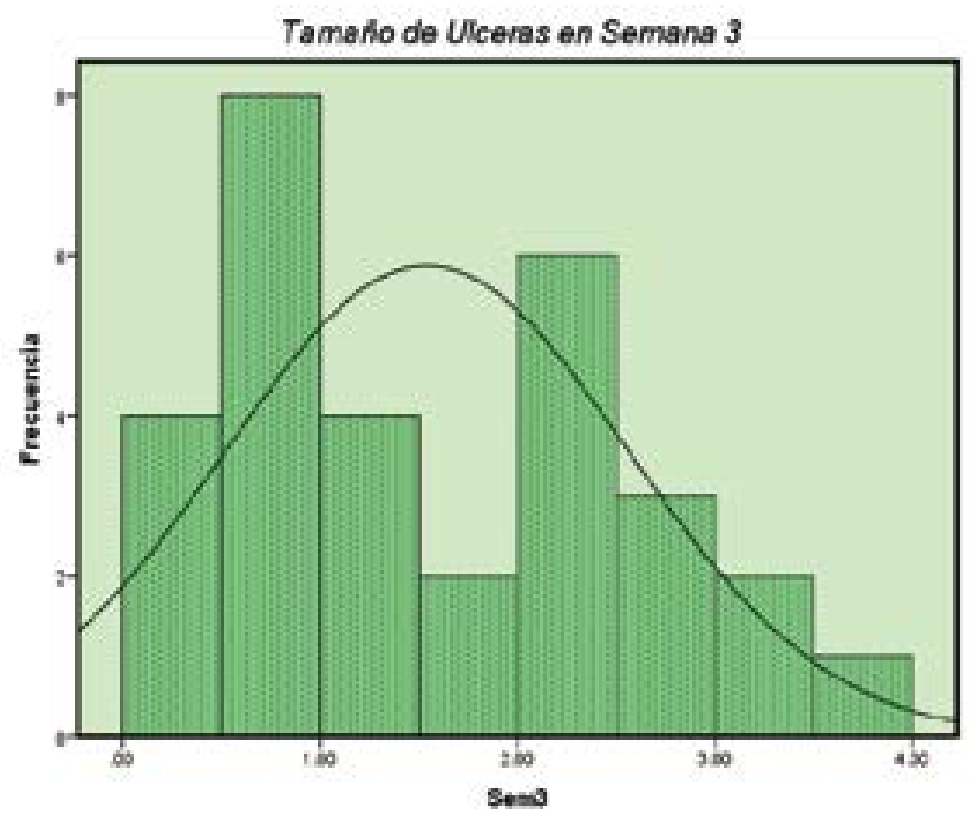

Figura 4. Histograma de distribución de los datos obtenidos de las mediciones realizadas la tercera semana

En la tercera semana se puede observar claramente la distribución y la tendencia de los datos, las medidas no superan a los 4 centímetros y se observa la acumulación hacia el cero. 


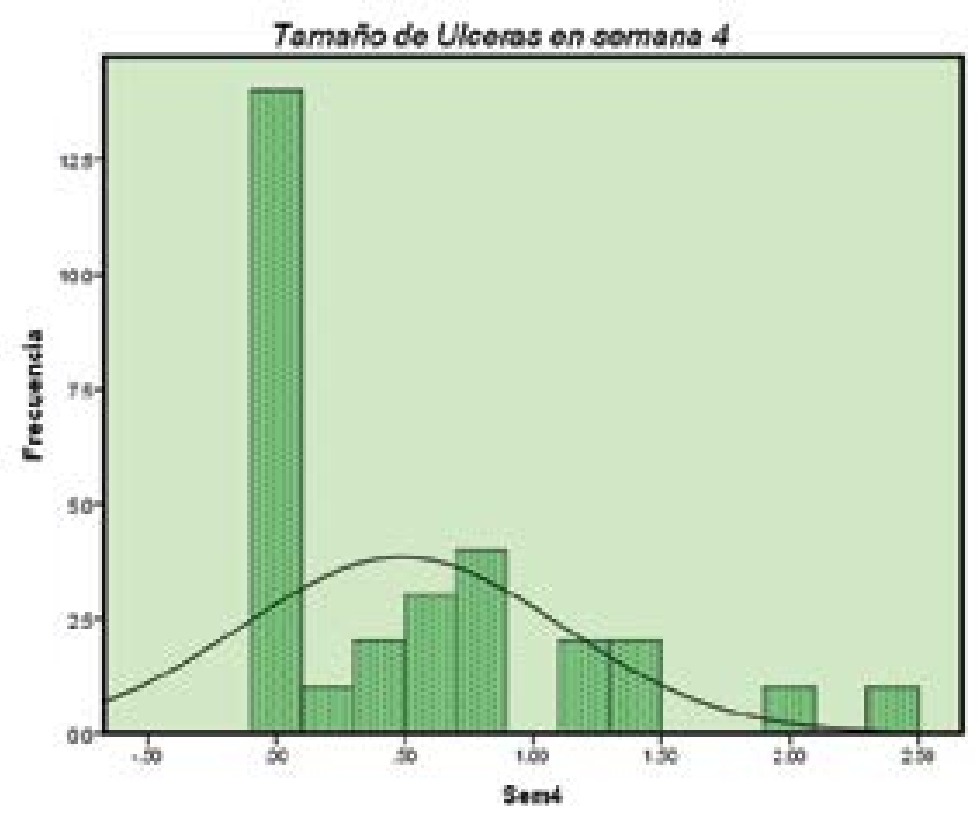

Figura 5. Histograma de distribución de los datos obtenidos de las mediciones realizadas la cuarta semana

En la cuarta y última semana de tratamiento apreciamos que los datos tienen una gran acumulación en cero y sólo muy pocos poseen entre 0,5 a 2,5 centímetros.

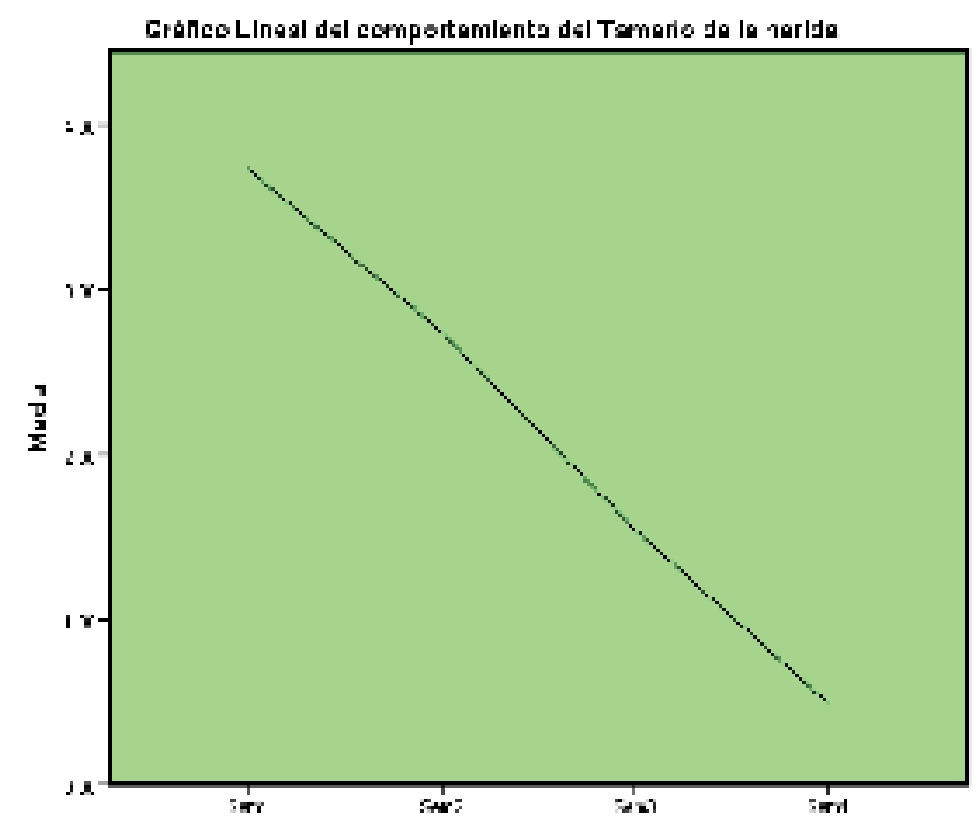

Figura 6. Gráfico lineal en el cual se observa el comportamiento de los datos durante las cuatro semanas, evidentemente el tamaño de la herida se redujo para toda la población. 


\section{ANÁLISIS Y DISCUSIÓN}

Los resultados obtenidos, muestran una marcada acción favorable de parte del gel (figura 1) formulado a base del extracto hidroalcohólico del fruto de la papaya (Carica papaya L.), en el proceso de cicatrización y regeneración del tejido comprometido por la úlcera por presión

(Tablas 1, 4, 5 y 6) (Fig 2, 3, 4, 5 y 6).

Durante la evaluación diaria, se observó que no hubo alergias en el sitio de aplicación ni complicaciones visibles por lo que se puede decir, en términos generales, que el ensayo tuvo una evolución satisfactoria. La marcha fitoquímica mostró que el extracto contiene una cantidad importante de Flavonoides, cuyo efecto antiinflamatorio es muy bien conocido; así mismo, los taninos que contiene le confiere al extracto propiedades astringentes y antiinflamatorias. El estudio de estabilidad acelerado (Tabla 3) practicado al gel, demostró que éste es estable y perfectamente compatible con el extracto, no evidenciándose cambios organolépticos ni fisicoquímicos, por lo que se puede afirmar que tendría un tiempo de vida media de tres años.

Es conocido que, en forma de medicamento natural,lapapaínaseutilizacomoantiinflamatorio enzimático proteolítico útil en el control de hematomas, inflamación y edemas producidos por lesiones traumáticas o antiinflamatorias ${ }^{(7)}$. Es ablandador de carnes, cicatrizante y es apreciada por sus propiedades suavizantes, hidratantes, regenerativas y exfoliantes y por su importante contenido de carotenos, precursores de vitamina A y por la actividad como antirradicales libres de los mismos ${ }^{(8)}$

El estudio farmacológico, llevado a cabo con 35 voluntarios dejó la evidencia que el extracto hidroalcohólico del fruto de la papaya administrado en forma de gel (Fig 1), tiene efecto en el proceso de regeneración del tejido dañado, debido posiblemente al efecto sinérgico de la enzima papaína con los metabolitos flavonoides + taninos.

\section{CONCLUSIONES}

1. El extracto hidroalcohólico del fruto de la papaya (Carica papaya L.), administrado en forma de gel, mostró tener efecto en el proceso de cicatrización y regeneración del tejido comprometido por la úlcera por presión.

2. De acuerdo con la escala calificadora de Balbuena Gonzáles y Chapano Jairo, el $60,00 \%$ fue evaluado como excelente, el $25,00 \%$ como bueno y el $15,00 \%$ como regular y el $0,00 \%$ como nulo.

3. Por su fácil adquisición, bajo costo y ser una sustancia de origen natural, el extracto hidroalcohólico del fruto de la papaya (Carica papaya L.) , puede constituir un importante activo para su aplicación en el tratamiento de úlceras por presión de estadío I y II.

\section{REFERENCIAS BIBLIOGRÁFICAS}

1. GARCÍA, F; PANCORBO, P; TORRA, J; (2004). Úlceras por presión en el paciente crítico. Documento obtenido de: http://www.enfermeria21.com/Generalitats/ficheros/verFichero. php?NzAwNDEzOTU\%3D

2. SERVICIO ANDALUZ DE SALUD (2007) Guía de práctica clínica para la prevención y el tratamiento de las úlceras por presión. Servicio Andaluz de Salud. Obtenido de guía PDF: http://www.juntadeandalucia.es/servicioandaluzdesalud/publicaciones/listadodeterminado. asp?idp $=283$

3. HOSPITAL GENERAL UNIVERSITARIO - VALENCIA. Guía de práctica clínica en la prevención de úlceras por presión (Unidad de enfermería dermatológica, úlceras y heridas).. 
Obtenido de: http://chguv.san.gva.es/Descargas/AreaEnfermeria/AreaProcsAmbu/UCDermatologicos/Guia_Prev_Ulceras_Pres.pdf

4. MARTINEZ, R. (1992). Guía de práctica clínica en prevención de úlceras por presión (Unidad de enfermería dermatológica, úlceras y heridas) H.U. San Carlos de Madrid, Valencia. Obtenido de: http://chguv.san.gva.es/Descargas/AreaEnfermeria/AreaProcsAmbu/UCDermatologicos/Guia_Prev_Ulceras_Pres..pdf

5. LÓPEZ, P. (2005). Enfermero. Responsable Unidad Interdisciplinar de Heridas Crónicas. Grupo Nacional para el Estudio y Asesoramiento en Úlceras por Presión y Heridas Crónicas. Agencia Valenciana de Salud. ESPAÑA

6. BRITISH HERBAL PHARMACOPOEIA. (1990) Vol. I. Bournemouth, Dorset: British Herbal Medical Association, pp. 69-70.

7. BÉZANGER-BEAUQUESNE, L; PINKAS, M; TORCK, M. (1986). Les Plantes dans la Therapeutique Moderne. $2^{\circ}$.Paris: Maloine, pp.320-1.

8. VAN HELLEMONT, J.(1986). Compendium de Phytotherapie. Bruxelles: Association Pharmaceutique Belge, pp. 38-40. 